\title{
Healthcare Resource Utilization among Non-Valvular Atrial Fibrillation Patients Who Switched from Warfarin to a Novel Oral Anti-Coagulant
}

\author{
Jessica Franchino-Elder', Adrienne Gilligan'2, Xue Song'2*, Briain O Hartaigh', Caroline Henriques ${ }^{2}$, Amy \\ Sainski-Nguyen ${ }^{2}$, Cheng Wang' \\ 'Boehringer-Ingelheim Pharmaceuticals, Inc., Ridgefield, CT, USA \\ 2IBM Watson Health, Cambridge, MA, USA
}

Article Info

\section{Article Notes}

Received: August 21, 2019

Accepted: September 9, 2019

\section{*Correspondence:}

${ }^{*}$ Dr. Xue Song, PhD, IBM Watson Health, Life Sciences, 75 Binney Street,Cambridge, MA 02142, USA; Telephone No: +1508-842-5312; Email: songx@us.ibm.com.

(C) 2019 Song $X$. This article is distributed under the terms of the Creative Commons Attribution 4.0 International License.

\section{Keywords:}

Atrial fibrillation

Novel oral anticoagulants

Healthcare resource Utilization

Warfarin

Dabigatran

Apixaban

Rivaroxaban

\section{Abstract}

Among patients with non-valvular atrial fibrillation (NVAF), switching from warfarin to novel oral anticoagulants (NOACs) is common, yet clarifying the differences in the effect of NOACs on all-cause healthcare resource utilization (HCRU) are unknown. Adult NVAF patients who switched from warfarin to dabigatran, apixaban, or rivaroxaban were identified in MarketScan databases between 10/2010-12/2015. Patients had 12 months pre-period (index date was 1st NOAC claim) and were followed up to 12 months until medication discontinuation, end of enrollment, inpatient death, or 12/2016. Overall, 8,679 and 5,761 dabigatran switchers were matched (1:1) to rivaroxaban and apixaban switchers (mean age 73-74 years). Compared with rivaroxaban switchers, a lower proportion of dabigatran switchers had an inpatient (IP) visit $(20.0 \%$ vs. $21.6 \%, p=0.008)$. Dabigatran switchers had lower per-patient-per-month (PPPM) total outpatient ( 3.87 vs. $4.06, p=0.002$ ), emergency department (ED; 0.48 vs. $0.52, p=0.026)$, outpatient office (1.17 vs. $1.22, p<0.001)$, and other outpatient ( 2.71 vs. $2.83, p=0.043$ ) visits compared with rivaroxaban switchers. A similar proportion of dabigatran and apixaban switchers had an IP visit (20.7\% vs. $21.2 \%)$; compared with apixaban switchers, dabigatran switchers had significantly more PPPM IP visits ( 0.23 vs. $0.21, p=0.031$ ) but significantly lower ED visits ( 0.47 vs. $0.52, p=0.016$ ). Post-discharge 30 -day readmission rates were comparable among warfarin-to-NOAC switching groups. Time to readmission was longer for dabigatran versus rivaroxaban switchers (8.2 vs. 7.8 days, $p<0.001$ ), but comparable with apixaban patients ( 8.1 vs. 8.4 days). Switching to dabigatran after warfarin discontinuation may lower HCRU among NVAF patients compared with switching to rivaroxaban or apixaban.

\section{Introduction}

Atrial fibrillation (AF) is estimated to affect 3 to 6 million people in the United States (US), with prevalence expected to double by 2030 , accounting for approximately $15 \%$ of all strokes ${ }^{1-6}$. Patients with $\mathrm{AF}$ are four to five times more likely to have a stroke than patients without $\mathrm{AF}^{2,3,7}$. Non-valvular atrial fibrillation (NVAF), defined as $\mathrm{AF}$ in the absence of mitral stenosis or valvular prostheses, accounts for $95 \%$ of all AF cases in the $\mathrm{US}^{2,8}$.

Anticoagulation therapy has been the standard of care for reducing risks of thromboembolic and ischemic stroke events in patients with AF going back many years ${ }^{9}$. Antithrombotic treatment guidelines in the US, Canada, and Europe recommend chronic oral anticoagulation (OAC) for patients with $\mathrm{AF}$, rather than no therapy, aspirin, or a combination therapy of aspirin with clopidogrel, especially for patients with an intermediate or high risk of stroke $\mathrm{e}^{9-14}$. Warfarin had historically been the most commonly prescribed 
OAC since its approval in 1954; however, recent US Food and Drug Administration approvals have made available four mechanistically novel oral anticoagulants (NOACs), including the direct thrombin inhibitor dabigatran (approved October 2010), and factor Xa inhibitors rivaroxaban, apixaban, and edoxaban (approved in July 2011, October 2012, and January 2015, respectively) for prophylaxis of ischemic stroke and other complications ${ }^{10,13}$. To this end, the recently revised American Heart Association (AHA)/American College of Cardiology (ACC)/ Heart Rhythm Society (HRS) guidelines advocate the use of NOACs over warfarin ${ }^{15}$.

Since dabigatran's approval, several real-world clinical practice studies have evaluated the associated clinical outcomes, healthcare utilization, costs, and treatment patterns, mostly with warfarin as the comparator ${ }^{16-18}$. A study of the RE-LY trial comparing the effectiveness of dabigatran versus warfarin found dabigatran superior in reducing the risk of both stroke and systemic embolism ${ }^{19}$. While a large proportion of newly diagnosed AF patients initiate OAC therapy with warfarin, many of them may discontinue warfarin due to regular monitoring of International Normalized Ratio, interactions of warfarin with food, alcohol, and other drugs, complications, new comorbidities, and certain genetic variations that may predispose patients to adverse events ${ }^{15,20,21}$. To date, there is limited real-world data available assessing differences between NOACs for those who switched from warfarin. The primary objective of this study was to compare allcause healthcare resource utilization of warfarin-treated patients diagnosed with NVAF who switched to dabigatran, rivaroxaban, or apixaban.

\section{Objectives}

The objective of this study was to compare allcause healthcare resource utilization among patients diagnosed with NVAF who were treated with warfarin and subsequently switched to dabigatran, rivaroxaban, or apixaban in a head-to-head comparison using a large realworld data source.

\section{Methods}

\section{Study design and data source}

This retrospective analysis of patients diagnosed with AF compared healthcare resource utilization using real-world healthcare claims data for patients in the US with commercial and/or Medicare supplemental insurance coverage treated initially with warfarin and who subsequently switched to dabigatran, rivaroxaban, or apixaban. All study data was obtained from de-identified health plan enrollment records, inpatient and outpatient medical claims and outpatient prescription claims using International Classification of Diseases, 9th and 10th Revision, Clinical Modification (ICD-9-CM and ICD-10-CM) codes, Current Procedural Terminology 4th edition (CPT4) codes, Healthcare Common Procedure Coding System (HCPCS) codes, and National Drug Codes (NDCs).

The data source was administrative healthcare claims data from the 2009-2016 MarketScan $®$ Commercial Claims and Encounters (commercial) Database and Medicare Supplemental and Coordination of Benefit (Medicare) Database (IBM Watson Health, Cambridge, MA). These databases contain the complete longitudinal records of inpatient services, outpatient services, and prescription drug claims for commercially-insured and Medicareeligible patients covered under a variety of health plans, including dates of service, places of service, and all payments. All database records are de-identified and fully compliant with US patient confidentiality requirements set forth in Sections 164.514 (a)-(b)1ii of the Health Insurance Portability and Accountability Act (HIPAA) regarding the determination and documentation of statistically deidentified data. Because this study used only de-identified patient records and did not involve the collection, use, or transmittal of individually identifiable data, Institutional Review Board approval to conduct this study was not necessary.

\section{Subject selection}

Patients were selected for the study if they had an inpatient or outpatient medical claim with at least one diagnosis code for AF (ICD-9-CM diagnosis code 427.31 or ICD-10-CM diagnosis codes I480, I481, I482, or I4891) in any position between October 1, 2010 and December 31, 2015 (patient selection period) and at least one outpatient pharmacy claim during that period for warfarin on or after the first observed AF diagnosis and prior to initiation of dabigatran, rivaroxaban, or apixaban. Patients were further required to have at least one outpatient pharmacy claim for dabigatran, rivaroxaban, or apixaban after a pharmacy claim for warfarin. The inclusion dates for patients receiving these three NOACs depended on the drugs' launch dates, as follows: dabigatran 10/01/2010$12 / 31 / 2015$, rivaroxaban $11 / 01 / 2011-12 / 31 / 2015$, and apixaban 12/01/2012-12/31/2015. The date of the first pharmacy claim for dabigatran, rivaroxaban, or apixaban was the patient's index date.

All patients were required to be continuously enrolled for 12 months prior to the index date (pre-index period) and for up to 12 months following the index date (followup period). Patients were required to be at least 18 years of age on their index date.

Patients were excluded if they had an inpatient or outpatient medical claim that included any of the following procedures or diagnoses indicating possible valvular disease within 6 months prior to the first observed $\mathrm{AF}$ diagnosis: cardiac surgery, hyperthyroidism, myocarditis, 
pericarditis, pulmonary embolism, valve replacement, chronic rheumatic heart disease, or valvular disease, and for evidence of pregnancy (codes available upon request). Patients were also excluded if they had pharmacy claims for an anticoagulant other than warfarin (apixaban, dabigatran, rivaroxaban, argatroban, dalteparin, edoxaban, enoxaparin, fondaparinux, heparin, or tinzaparin) in the 6-month pre-index period, if they did not discontinue warfarin during the follow-up period, or if they had been indexed on a $10 \mathrm{mg}$ dose of rivaroxaban.

The follow-up period was a variable-length up to 12 months from the index date until the earliest evidence of switching to a different NVAF medication, discontinuation, inpatient death, end of continuous enrollment, or study end (December 31, 2016). Healthcare resource utilization was reported per patient per month (PPPM) to account for the variable length of follow-up periods ${ }^{22-24}$.

Switching was defined as the presence of a different anticoagulant (apixaban, dabigatran, edoxaban, rivaroxaban, warfarin, argatroban, dalteparin, enoxaparin, fondaparinux, heparin, or tinzaparin) within 30 days of the end of the days' supply of the index medication and evidence of discontinuing the index medication (definition below).

Discontinuation was defined as the lack of subsequent claims for the index medication beyond 90 days following the exhaustion of the previous claim's days' supply. The discontinuation date was the last day of supply immediately preceding the $>90$-day gap.

\section{Matching}

To control for demographic and clinical characteristics that could potentially affect the interpretation of study outcomes in head-to-head comparisons, propensity score matching was conducted to match apixaban and rivaroxaban patients to dabigatran patients. Matching factors included age, payer (commercial vs. Medicare), sex, geographic region of residence, health plan type, baseline comorbidities, stroke risk scores, bleeding risk scores, baseline total costs, and baseline medication use (beta blocker, calcium channel blocker, diuretics, other antihypertensives, antihyperlipidemics, steroids, anti-diabetic medications, antiarrhythmics, antiplatelets, non-steroidal anti-inflammatory drugs, selective serotonin reuptake inhibitors, and serotonin-norepinephrine reuptake inhibitors). Propensity scores were generated using a series of logistic regression models to predict the probability that a patient who discontinued warfarin switched to dabigatran vs. rivaroxaban and dabigatran vs. apixaban based on observed characteristics. Once each patient was assigned a propensity score, dabigatran switchers were matched 1:1 against the available pools of rivaroxaban and apixaban patients using the nearest neighbor matching technique without replacement and allowing a caliper of $1 / 4$ of the standard deviation of estimated propensity scores. To examine the quality of the match, standardized differences were calculated before and after the matching, with an absolute value less than 10 indicating an acceptable match.

\section{Outcome measures}

All-cause healthcare utilization was reported overall and by type of service: inpatient, emergency department (ED), outpatient, physician office visits, other outpatient services, and pharmacy. The rate for all outcomes was calculated across all patients, regardless if they had a specific service, and among patients with at least one service. Healthcare resource utilization was reported on a PPPM basis to account for variable length of follow-up by dividing the total utilization over the observation period by the number of months of the observation period, and then averaged across patients to obtain PPPM.

Hospital length of stay (LOS) was reported in the follow-up period as the total number of inpatient days in the follow-up period divided by the number of inpatient admissions. Cumulative LOS (bed days) was reported as the total number of inpatient days in the follow-up period.

Readmissions within 30 days following discharge were evaluated among patients with at least 30 days of followup post discharge. The payment mechanism for Medicare Part A causes the majority of those patients to be missed using standard readmission methodology; therefore, a published algorithm was utilized to help identify Medicare readmissions ${ }^{25}$.

\section{Key explanatory variables}

Demographic variables measured on the index date included age in years, sex (male or female), payer type (commercial or Medicare), health plan type, United States Census Bureau geographic region of residence (Northeast, North Central, South, West), physician specialty on the index claim, duration of post-index period, and days between the index drug's launch date and index. Total costs were measured in the 12-month pre-index period.

Clinical characteristics measured during the 12-month pre-index period included the following: General measure of overall health status included the Deyo-Charlson Comorbidity Index (DCI), the number of unique medications, number of inpatient visits observed, and number of physician visits observed ${ }^{26}$. Specific comorbidities included chronic kidney disease, chronic obstructive pulmonary disease, pneumonia, cirrhosis, hepatitis, coronary artery disease, diabetes mellitus, gastrointestinal (GI) bleeding, heart failure, ischemic stroke, transient ischemic attacks (TIAs), hemorrhagic stroke, intracranial bleeding, extracranial bleeding, myocardial infarction (MI - acute 
and/or old), paraplegia/hemiplegia, psychiatric disorders, and venous thromboembolism. Stroke risk was assessed using the $\mathrm{CHADS}_{2}$ and $\mathrm{CHA}_{2} \mathrm{DS}_{2}$-VASc scores, based on the presence of specified diagnoses from inpatient and outpatient claims in the 12 -month pre-index period ${ }^{27}$. $\mathrm{CHADS}_{2}$ score is calculated based on Congestive heart failure, Hypertension, Age, Diabetes, and Stroke/transient ischemic stroke. The $\mathrm{CHA}_{2} \mathrm{DS}_{2}$-VASc score adds vascular disease and female, has different points for two age groups, and includes all the other components of $\mathrm{CHADS}_{2}$. Bleeding risk was assessed using the HAS-BLED and ATRIA scores, also based on specified diagnoses in inpatient and outpatient claims during the 12-month pre-index period ${ }^{28}$. HAS-BLED is based on evidence of hypertension, abnormal renal or liver function, stroke, major bleeding, labile international normalized ratio (INR), elderly, and drug or alcohol use. Components of ATRIA are anemia, severe renal disease, age, hemorrhagic diagnosis, and hypertension.

International Normalized Ratio (INR) testing was determined via claims with a CPT or HCPCS procedure code. In addition, the following timings were measured: days from the first AF diagnosis to warfarin exposure, days from the first AF diagnosis to index drug initiation, days from the exhaustion of the days' supply of the last warfarin claim to index drug initiation, and the length of warfarin therapy.

Use of medications in the following medication classes during the 12-month pre-index period were recorded: beta blockers, calcium channel blockers, diuretics, other antihypertensives, antihyperlipidemics, corticosteroids, antidiabetics, antiarrhythmic (amiodarone, propafenone, flecainide, dronedarone, sotalol, dofetilide, disopyramide, quinidine), antiplatelets, nonsteroidal anti-inflammatory drugs, selective serotonin reuptake inhibitors, and serotonin-norepinephrine reuptake inhibitors ${ }^{29}$.

\section{Statistical analysis}

Categorical variables are presented as counts and percentages. Continuous variables are summarized by providing means and standard deviations. Statistical tests of significance are conducted for evaluating differences between rivaroxaban or apixaban cohorts with respective matched dabigatran cohorts using Chi-square tests for categorical variables, and t-tests for continuous variables. A critical value of $\mathrm{p}<0.05$ was specified a priori as the threshold for statistical significance. Data management, analytic file building, and statistical analyses were conducted using SAS software, version 9.4 (SAS Institute Inc, Cary, North Carolina).

\section{Results}

The final matched cohorts meeting all selection criteria included 8,679 rivaroxaban patients and 5,761 apixaban patients, each matched 1:1 with an equal number of dabigatran patients (Figure 1). Mean ages ranged 73.1-74.4 years, with $56.2 \%-58.8 \%$ males (Table 1 ). Most patients were Medicare-eligible retirees (76.2\%-78.6\%), under preferred provider (45.0\%-47.5\%) or comprehensive/ indemnity (36.8\%-38.9\%) arrangements. The most common physician specialty on the index claim was cardiologist (32.6\%-33.0\%). Mean follow-up was shorter for dabigatran patients compared with apixaban (225 vs. 233 days, $\mathrm{p}<0.001$ ) but comparable with rivaroxaban patients (228 vs. 231 days, $p=0.123$ ). There were otherwise no significant demographic differences between comparison cohorts. While $\mathrm{p}$ values found significantly lower baseline total expenditures for dabigatran cohorts compared with both rivaroxaban patients $(\$ 29,624$ vs. $\$ 34,321 ; \mathrm{p}<0.001)$ and apixaban patients $(\$ 32,275$ vs. $\$ 40,124 ; \mathrm{p}<0.001)$, standardized differences ( 6 for dabigatran vs. rivaroxaban and 9 for dabigatran vs. apixaban) suggest that baseline costs were well balanced between the comparison cohorts.

Nearly all baseline clinical characteristics were similar between the comparison cohorts (Table 2). Mean values of DCI scores were 1.8 for dabigatran vs. rivaroxaban and 1.9 for dabigatran vs. apixaban patients. Patients had 12.2-12.9 unique medications pre-index, made 0.6 inpatient visits, and had 13.5-14.1 physician office visits. The most common baseline comorbidities were coronary artery disease (38.2\%-43.4\%), heart failure (26.6\%-31.4\%), diabetes mellitus (28.6\%-30.1\%), followed by psychiatric disorders, chronic obstructive pulmonary disease, and chronic kidney disease. Extracranial bleeding was more common (15.6\%$19.2 \%)$ than gastrointestinal $(6.0 \%-8.1 \%)$ or intracranial bleeding (1.2\%-1.6\%). Pre-index medications received by most patients included beta blockers (72.9\%-75.3\%), antihyperlipidemics (63.6\%-66.0\%) and diuretics (55.1\%$57.2 \%$ ), with similar rates among comparator cohorts.

Mean days from AF diagnosis to index medication initiation were significantly shorter for dabigatran than for either rivaroxaban (339 vs. 790 days, $\mathrm{p}<0.001$ ) or apixaban (358 vs. 1065 days, $\mathrm{p}<0.001$ ), owing to a combination of shorter days from AF diagnosis to warfarin initiation, shorter length of warfarin therapy, and shorter time from the end of warfarin therapy to index drug initiation (Table 2).

The proportion of patients with at least one inpatient admission was lower for dabigatran compared with rivaroxaban $(20.0 \%$ vs. $21.6 \%, \mathrm{p}=0.008)$, but similar between dabigatran and apixaban patients $(20.7 \%$ vs $21.2 \%, \mathrm{p}=0.522$ ) (Figure 2). Hospital LOS and cumulative days in hospitals were similar among all three cohorts (0.91.1 and 1.2-1.3) (Table 3). The percentage of patients with at least one ED visit was lower for dabigatran compared with rivaroxaban $(27.4 \%$ vs. $29.7 \%, \mathrm{p}<0.001)$, but similar between dabigatran and apixaban patients $(28.3 \%$ vs $29.8 \%, p=0.074$ ). Significantly fewer dabigatran patients had claims for other outpatient services versus either 
Patients with $\geq 1$ non-diagnostic claim with diagnosis code for atrial fibrillation ${ }^{\mathrm{a}}(\mathrm{AF})$ in any position between 10/01/2010 and 12/31/2015.

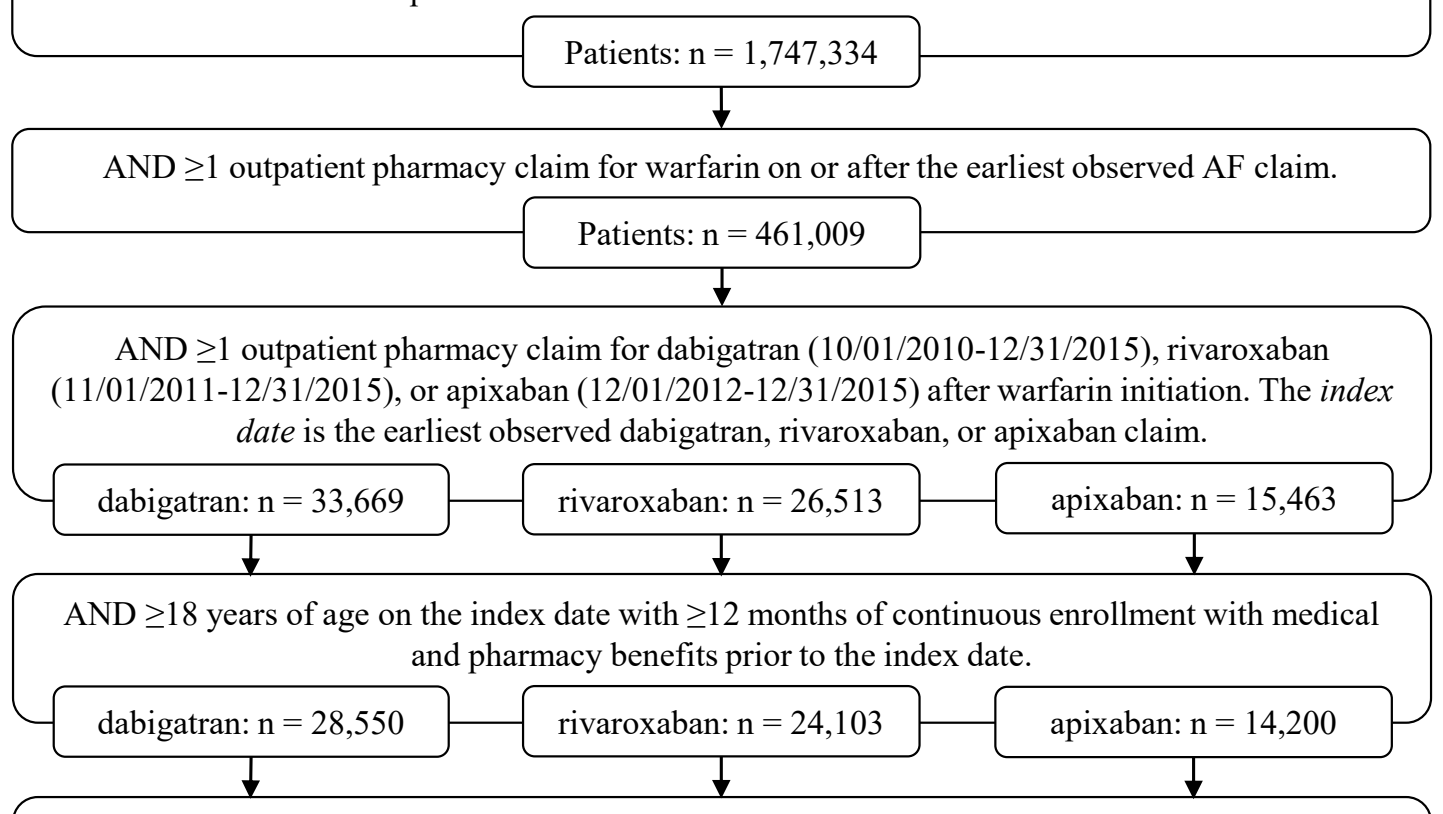

AND no outpatient pharmacy claim for any oral anticoagulant except warfarin in the 6 months prior to the index date.

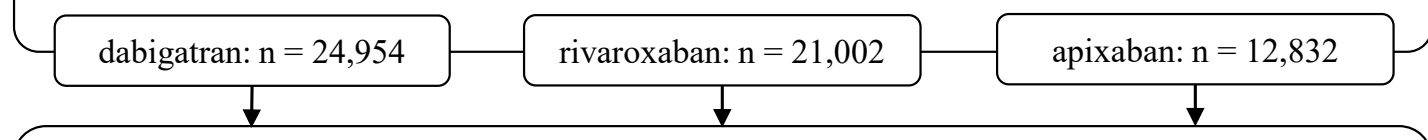

AND no evidence of cardiac surgery, hyperthyroidism, myocarditis, pericarditis, pregnancy, pulmonary embolism, valve replacement, valvular heart disease, or chronic rheumatic heart disease during the 6 months prior to the earliest AF diagnosis.

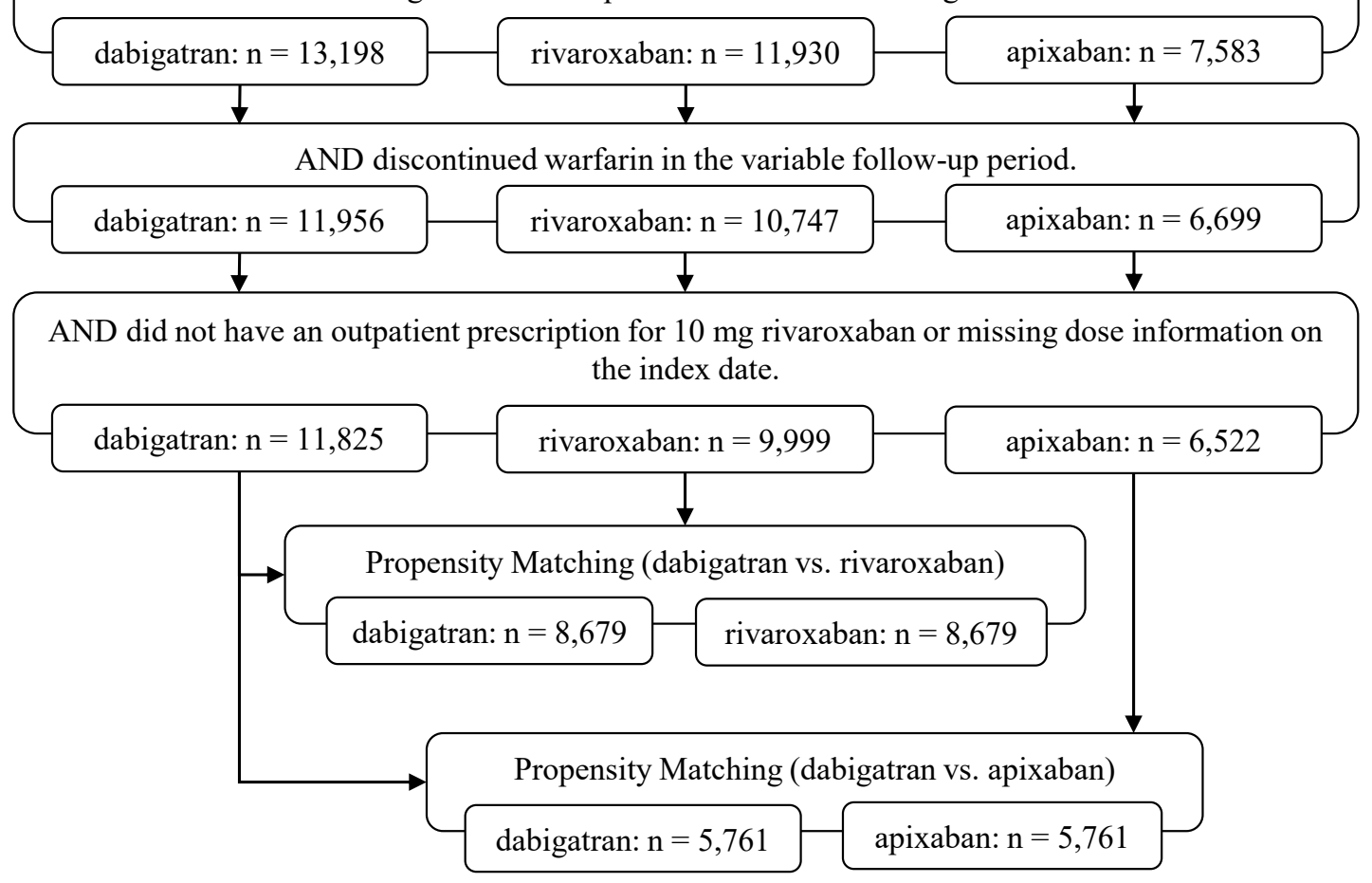

Note: ${ }^{a}$ ICD-9-CM diagnosis code 427.31 and ICD-10-CM diagnosis codes 148.0, 148.1, 148.2, and 148.91. ICD-9/10-CM, International Classification of Diseases, Ninth/Tenth Revision, Clinical Modification.

Figure 1: Patient attrition. 
Table 1: Baseline patient demographic characteristics

\begin{tabular}{|c|c|c|c|c|c|c|c|c|}
\hline Demographics & Dabigatran & Apixaban & $P$ value & Std Diff & Dabigatran & Rivaroxaban & $P$ value & Std Diff \\
\hline Patient count & 5,761 & 5,761 & & & 8,679 & 8,679 & & \\
\hline Age (mean, SD) & 74.1 (11.2) & $74.4(11.3)$ & 0.106 & 0.066 & $73.1(11.3)$ & $73.3(11.3)$ & 0.229 & 0.095 \\
\hline \multicolumn{9}{|l|}{ Age group (\%) } \\
\hline $18-44$ & $0.8 \%$ & $0.9 \%$ & 0.911 & 0.368 & $1.3 \%$ & $1.2 \%$ & 0.553 & 0.213 \\
\hline $45-54$ & $4.2 \%$ & $3.9 \%$ & 0.478 & 0.295 & $4.7 \%$ & $4.7 \%$ & 1.000 & 0.196 \\
\hline $55-64$ & $17.2 \%$ & $17.3 \%$ & 0.844 & 0.275 & $18.6 \%$ & $18.3 \%$ & 0.598 & 0.115 \\
\hline $65-74$ & $23.2 \%$ & $22.7 \%$ & 0.580 & 0.732 & $24.2 \%$ & $24.1 \%$ & 0.958 & 0.143 \\
\hline $75-84$ & $35.2 \%$ & $35.5 \%$ & 0.740 & 0.933 & $35.2 \%$ & $35.4 \%$ & 0.763 & 0.272 \\
\hline $85+$ & $19.5 \%$ & $19.7 \%$ & 0.778 & 0.173 & $16.1 \%$ & $16.3 \%$ & 0.726 & 0.934 \\
\hline \multicolumn{9}{|l|}{ Sex (\%) } \\
\hline Male & $56.7 \%$ & $56.2 \%$ & 0.573 & 0.596 & $58.8 \%$ & $58.0 \%$ & 0.317 & 0.968 \\
\hline Female & $43.3 \%$ & $43.8 \%$ & 0.573 & 0.596 & $41.2 \%$ & $42.0 \%$ & 0.317 & 0.968 \\
\hline \multicolumn{9}{|l|}{ Payer (\%) } \\
\hline Commercial & $21.5 \%$ & $21.4 \%$ & 0.910 & 0.108 & $23.8 \%$ & $23.5 \%$ & 0.592 & 0.008 \\
\hline Medicare & $78.5 \%$ & $78.6 \%$ & 0.910 & 0.108 & $76.2 \%$ & $76.5 \%$ & 0.592 & 0.008 \\
\hline \multicolumn{9}{|l|}{ Health plan type (\%) } \\
\hline Comprehensive/indemnity & $38.1 \%$ & $38.9 \%$ & 0.389 & 0.684 & $36.8 \%$ & $37.4 \%$ & 0.460 & 0.101 \\
\hline EPO/PPO & $45.4 \%$ & $45.0 \%$ & 0.667 & 0.356 & $47.5 \%$ & $47.4 \%$ & 0.952 & 0.581 \\
\hline POS & $5.1 \%$ & $4.9 \%$ & 0.607 & 0.447 & $5.3 \%$ & $5.1 \%$ & 0.494 & 0.569 \\
\hline $\mathrm{HMO}$ & $6.6 \%$ & $6.5 \%$ & 0.792 & 0.921 & $6.3 \%$ & $6.2 \%$ & 0.802 & 0.391 \\
\hline CDHP/HDHP & $3.4 \%$ & $3.2 \%$ & 0.676 & 0.624 & $2.4 \%$ & $2.3 \%$ & 0.841 & 0.195 \\
\hline Other/unknown & $1.4 \%$ & $1.5 \%$ & 0.695 & 0.127 & $1.7 \%$ & $1.6 \%$ & 0.511 & 0.765 \\
\hline \multicolumn{9}{|l|}{ Geographic region (\%) } \\
\hline Northeast & $23.2 \%$ & $23.0 \%$ & 0.877 & 0.392 & $20.9 \%$ & $21.1 \%$ & 0.780 & 0.385 \\
\hline North Central & $26.8 \%$ & $26.6 \%$ & 0.850 & 0.235 & $26.7 \%$ & $27.0 \%$ & 0.572 & 0.244 \\
\hline South & $34.7 \%$ & $35.6 \%$ & 0.301 & 0.956 & $35.7 \%$ & $35.1 \%$ & 0.446 & 0.951 \\
\hline West & $15.2 \%$ & $14.6 \%$ & 0.333 & 0.426 & $16.2 \%$ & $16.2 \%$ & 0.951 & 0.563 \\
\hline Unknown & $0.3 \%$ & $0.3 \%$ & 1.000 & 0.126 & $0.6 \%$ & $0.6 \%$ & 0.762 & 0.695 \\
\hline \multicolumn{9}{|l|}{ Physician specialty on index claim (\%) } \\
\hline Internist & $22.5 \%$ & $23.0 \%$ & 0.534 & 0.072 & $22.1 \%$ & $23.2 \%$ & 0.082 & 0.000 \\
\hline Primary care & $19.8 \%$ & $21.6 \%$ & 0.023 & 0.010 & $20.3 \%$ & $21.4 \%$ & 0.062 & 0.029 \\
\hline Cardiologist & $32.7 \%$ & $32.7 \%$ & 0.937 & 0.193 & $32.6 \%$ & $33.0 \%$ & 0.497 & 0.022 \\
\hline Other specialist & $22.4 \%$ & $20.5 \%$ & 0.012 & 0.007 & $22.5 \%$ & $20.1 \%$ & $<0.001$ & 0.000 \\
\hline Unknown & $2.6 \%$ & $2.3 \%$ & 0.228 & 0.445 & $2.6 \%$ & $2.2 \%$ & 0.111 & 0.679 \\
\hline \multicolumn{9}{|l|}{ Reason for end of follow-up (\%) } \\
\hline Death in hospital & $0.8 \%$ & $1.1 \%$ & 0.122 & 0.490 & $0.6 \%$ & $1.0 \%$ & 0.014 & 0.661 \\
\hline End of continuous enrollment & $14.6 \%$ & $34.3 \%$ & $<0.001$ & 0.000 & $14.0 \%$ & $22.7 \%$ & $<0.001$ & 0.000 \\
\hline Anticoagulant switch & $17.6 \%$ & $6.7 \%$ & $<0.001$ & 0.000 & $17.1 \%$ & $10.9 \%$ & $<0.001$ & 0.000 \\
\hline Discontinuation of index Medication & $20.6 \%$ & $15.8 \%$ & $<0.001$ & 0.000 & $20.6 \%$ & $19.5 \%$ & 0.063 & 0.000 \\
\hline One year follow-up & $46.5 \%$ & $42.2 \%$ & $<0.001$ & 0.007 & $47.7 \%$ & $46.0 \%$ & 0.023 & 0.001 \\
\hline Launch date to index date, days (mean, SD) & $525(445)$ & $806(370)$ & $<0.001$ & $<0.001$ & $502(419)$ & $812(425)$ & $<0.001$ & 0.017 \\
\hline Days of post-index period (mean, SD) & $224(145)$ & $233(134)$ & $<0.001$ & $<0.001$ & $228(144)$ & $231(140)$ & 0.123 & $<0.001$ \\
\hline
\end{tabular}

$\mathrm{CDHP} / \mathrm{HDHP}$, consumer directed/high deductible health plan; EPO/PPO, exclusive/preferred provider organization; HMO, health maintenance organization; POS, point of service; SD, standard deviation; Std Diff, standardized difference.

rivaroxaban $(92.5 \%$ vs. $93.4 \%, \mathrm{p}<0.05)$ or apixaban $(92.3 \%$ vs. $93.9 \%, \mathrm{p}<0.001)$. The mean number of inpatient admissions and pharmacy claims was similar for dabigatran versus rivaroxaban $(0.046$ vs. $0.048 ; 4.256$ vs. 4.249$)$ or apixaban ( 0.048 vs. 0.045 ; 4.385 vs. 4.376 ). Overall, outpatient and ED visits were less frequent among dabigatran patients as compared with either rivaroxaban ( 3.678 vs. 3.886 and 0.131 vs. 0.154 , both $p<0.001$ ) or apixaban (3.794 vs. $3.949, \mathrm{p}<0.05$ and 0.133 vs. 0.155 , $\mathrm{p}<0.01$ ) patients. In addition, dabigatran patients had significantly fewer outpatient office and other outpatient visit claims compared with rivaroxaban patients $(1.044$ vs. $1.094, \mathrm{p}<0.001$ and 2.504 vs. $2.638, \mathrm{p}<0.05$ ). The proportion of hospitalized patients who experienced a 30-day readmission following discharge were comparable between dabigatran and rivaroxaban patients $(21.1 \%$ vs. 
Table 2: Baseline patient clinical characteristics

\begin{tabular}{|c|c|c|c|c|c|c|c|c|}
\hline Clinical Characteristics & Dabigatran & Apixaban & $P$ value & Std Diff & Dabigatran & Rivaroxaban & $P$ value & Std Diff \\
\hline Patient count & 5,761 & 5,761 & & & 8,679 & 8,679 & & \\
\hline \multicolumn{9}{|l|}{ Measures of overall health status (mean, SD) } \\
\hline Deyo-Charlson Comorbidity Index & $1.9(1.9)$ & $1.9(2.2)$ & 0.556 & 0.251 & $1.8(1.9)$ & $1.8(2.0)$ & 0.518 & 0.626 \\
\hline Number of unique medications & $12.9(6.2)$ & $12.5(6.2)$ & $<0.001$ & 0.309 & $12.4(6.1)$ & $12.2(6.2)$ & 0.017 & 0.099 \\
\hline Number of pre-index hospitalizations & $0.6(0.8)$ & $0.6(0.9)$ & 0.575 & 0.055 & $0.6(0.8)$ & $0.6(0.8)$ & 0.738 & 0.148 \\
\hline Number of pre-index physician visits & $14.0(9.4)$ & $14.1(10.6)$ & 0.633 & 0.561 & $13.5(9.2)$ & $13.6(10.3)$ & 0.474 & 0.150 \\
\hline \multicolumn{9}{|l|}{ Presence of comorbid conditions (\%) } \\
\hline Coronary artery disease & $43.4 \%$ & $42.7 \%$ & 0.419 & 0.831 & $38.9 \%$ & $38.2 \%$ & 0.342 & 0.557 \\
\hline Heart failure & $31.4 \%$ & $31.1 \%$ & 0.748 & 0.594 & $27.2 \%$ & $26.6 \%$ & 0.392 & 0.930 \\
\hline Diabetes mellitus & $29.8 \%$ & $30.1 \%$ & 0.699 & 0.864 & $29.1 \%$ & $28.6 \%$ & 0.492 & 0.828 \\
\hline Psychiatric disorders & $25.5 \%$ & $25.6 \%$ & 0.915 & 0.360 & $21.8 \%$ & $22.1 \%$ & 0.660 & 0.194 \\
\hline Chronic obstructive pulmonary disease & $23.5 \%$ & $23.6 \%$ & 0.843 & 0.975 & $21.7 \%$ & $21.8 \%$ & 0.854 & 0.667 \\
\hline Chronic kidney disease & $16.0 \%$ & $15.4 \%$ & 0.398 & 0.619 & $11.0 \%$ & $11.1 \%$ & 0.923 & 0.444 \\
\hline Pneumonia & $9.8 \%$ & $9.6 \%$ & 0.683 & 0.732 & $9.0 \%$ & $9.2 \%$ & 0.598 & 0.921 \\
\hline Venous thromboembolism & $6.2 \%$ & $6.4 \%$ & 0.759 & 0.197 & $4.9 \%$ & $5.4 \%$ & 0.169 & 0.071 \\
\hline Old myocardial infarction & $4.2 \%$ & $3.4 \%$ & 0.022 & 0.080 & $3.6 \%$ & $3.2 \%$ & 0.129 & 0.220 \\
\hline Acute myocardial infarction & $4.0 \%$ & $4.7 \%$ & 0.068 & 0.555 & $3.4 \%$ & $3.6 \%$ & 0.458 & 0.631 \\
\hline Hemiplegia & $3.4 \%$ & $3.2 \%$ & 0.498 & 0.387 & $2.9 \%$ & $2.9 \%$ & 0.892 & 1.000 \\
\hline \multicolumn{9}{|l|}{ Stroke conditions (\%) } \\
\hline Ischemic stroke/transient ischemic attack & $12.1 \%$ & $11.4 \%$ & 0.247 & 0.669 & $10.5 \%$ & $10.2 \%$ & 0.550 & 0.950 \\
\hline Ischemic stroke & $8.2 \%$ & $7.0 \%$ & 0.014 & 0.169 & $7.2 \%$ & $7.1 \%$ & 0.723 & 0.482 \\
\hline Transient ischemic attack & $6.7 \%$ & $7.0 \%$ & 0.508 & 0.804 & $5.9 \%$ & $5.6 \%$ & 0.514 & 0.589 \\
\hline Hemorrhagic stroke & $0.9 \%$ & $1.2 \%$ & 0.199 & 0.163 & $0.8 \%$ & $1.0 \%$ & 0.338 & 0.466 \\
\hline \multicolumn{9}{|l|}{ Bleeding-related conditions (\%) } \\
\hline Extracranial bleed & $19.2 \%$ & $19.0 \%$ & 0.794 & 0.472 & $15.6 \%$ & $15.8 \%$ & 0.661 & 0.578 \\
\hline Gastrointestinal bleed & $7.4 \%$ & $8.1 \%$ & 0.197 & 0.450 & $6.0 \%$ & $6.1 \%$ & 0.703 & 0.259 \\
\hline Intracranial bleed & $1.3 \%$ & $1.6 \%$ & 0.245 & 0.151 & $1.2 \%$ & $1.2 \%$ & 0.779 & 0.588 \\
\hline \multicolumn{9}{|l|}{ Measures of stroke risk (mean, SD) } \\
\hline $\mathrm{CHADS}_{2}$ & $2.2(1.3)$ & $2.2(1.3)$ & 0.865 & 0.914 & $2.0(1.3)$ & $1.9(1.3)$ & 0.622 & 0.775 \\
\hline $\mathrm{CHA}_{2} \mathrm{DS}_{2}$-VASC & $3.5(1.9)$ & $3.5(1.9)$ & 0.246 & 0.643 & $3.2(2.0)$ & $3.2(2.0)$ & 0.753 & 0.748 \\
\hline \multicolumn{9}{|l|}{ Measures of bleeding risk (mean, SD) } \\
\hline HAS-BLED & $1.7(1.1)$ & $1.7(1.1)$ & 0.522 & 0.386 & $1.6(1.1)$ & $1.5(1.1)$ & 0.700 & 0.430 \\
\hline ATRIA & $2.5(2.0)$ & $2.6(2.1)$ & $<0.001$ & 0.101 & $2.1(1.9)$ & $2.2(2.0)$ & 0.004 & 0.310 \\
\hline \multicolumn{9}{|l|}{ INR testing } \\
\hline Presence of INR testing (\%) & $55.8 \%$ & $57.3 \%$ & 0.106 & 0.021 & $55.1 \%$ & $57.5 \%$ & 0.001 & 0.003 \\
\hline Number of INR tests (mean, SD) & $4.5(6.6)$ & $4.5(6.8)$ & 0.822 & 0.448 & $4.4(6.4)$ & $4.4(6.7)$ & 0.801 & 0.110 \\
\hline $\begin{array}{l}\text { NVAF diagnosis to warfarin start, days (mean, } \\
\text { SD) }\end{array}$ & 63 (139) & $151(293)$ & $<0.001$ & 0.848 & $60(130)$ & $116(228)$ & $<0.001$ & 0.998 \\
\hline Duration of warfarin therapy, days (mean, SD) & $263(289)$ & $734(580)$ & $<0.001$ & 0.341 & $250(272)$ & $559(466)$ & $<0.001$ & 0.069 \\
\hline Last warfarin claim to index, days (mean, SD) & $33(172)$ & $180(399)$ & $<0.001$ & 0.881 & $29(162)$ & $115(304)$ & $<0.001$ & 0.994 \\
\hline \multicolumn{9}{|l|}{ Warfarin gaps (\%) } \\
\hline$\leq 30$ days & $32.8 \%$ & $43.0 \%$ & $<0.001$ & 0.140 & $31.9 \%$ & $40.3 \%$ & $<0.001$ & 0.058 \\
\hline $31-60$ days & $7.9 \%$ & $10.2 \%$ & $<0.001$ & 0.000 & $7.8 \%$ & $11.1 \%$ & $<0.001$ & 0.684 \\
\hline $61-90$ days & $3.1 \%$ & $4.9 \%$ & $<0.001$ & 0.425 & $3.1 \%$ & $4.4 \%$ & $<0.001$ & 0.377 \\
\hline$>90$ days & $56.2 \%$ & $41.9 \%$ & $<0.001$ & 0.001 & $57.3 \%$ & $44.2 \%$ & $<0.001$ & 0.000 \\
\hline NVAF diagnosis to index, days (mean, SD) & $358(354)$ & $1065(553)$ & $<0.001$ & 0.379 & $339(332)$ & $790(478)$ & $<0.001$ & 0.109 \\
\hline \multicolumn{9}{|l|}{ Pre-index medications (\%) } \\
\hline Beta blockers & $75.3 \%$ & $75.3 \%$ & 0.966 & 0.231 & $73.5 \%$ & $72.9 \%$ & 0.337 & 0.672 \\
\hline Antihyperlipidemics & $65.8 \%$ & $66.0 \%$ & 0.798 & 0.602 & $63.9 \%$ & $63.6 \%$ & 0.613 & 0.393 \\
\hline Diuretics & $56.9 \%$ & $57.2 \%$ & 0.778 & 0.577 & $55.1 \%$ & $55.1 \%$ & 0.939 & 0.984 \\
\hline Corticosteroids & $43.4 \%$ & $43.0 \%$ & 0.638 & 0.853 & $42.4 \%$ & $42.8 \%$ & 0.591 & 0.889 \\
\hline Calcium channel blockers & $38.6 \%$ & $39.1 \%$ & 0.566 & 0.362 & $38.2 \%$ & $38.6 \%$ & 0.640 & 0.732 \\
\hline
\end{tabular}


Franchino-Elder J, Gilligan A, Song X, Hartaigh BO, Henriques C, Sainski-Nguyen A, Wang

C. Healthcare Resource Utilization among Non-Valvular Atrial Fibrillation Patients Who

Switched from Warfarin to a Novel Oral Anti-Coagulant. J Cardiol and Cardiovasc Sciences. 2019;3(4):36-46

\begin{tabular}{|l|c|c|c|c|c|c|c|c|}
\hline Other antihypertensives & $37.6 \%$ & $37.7 \%$ & 0.908 & 0.893 & $39.0 \%$ & $38.8 \%$ & 0.803 & 0.688 \\
\hline Antiarrhythmics & $31.9 \%$ & $32.3 \%$ & 0.646 & 0.637 & $30.4 \%$ & $30.2 \%$ & 0.766 & 0.915 \\
\hline Antidiabetic agents & $23.2 \%$ & $23.1 \%$ & 0.860 & 0.951 & $23.2 \%$ & $23.0 \%$ & 0.760 & 0.677 \\
\hline Selective serotonin reuptake inhibitors & $16.0 \%$ & $16.0 \%$ & 0.939 & 0.829 & $15.1 \%$ & $15.4 \%$ & 0.526 & 0.668 \\
\hline Non-steroidal anti-inflammatory drugs & $13.3 \%$ & $12.6 \%$ & 0.244 & 0.847 & $14.5 \%$ & $14.2 \%$ & 0.634 & 0.757 \\
\hline Antiplatelets & $10.3 \%$ & $9.9 \%$ & 0.404 & 0.695 & $9.3 \%$ & $9.1 \%$ & 0.674 & 0.664 \\
\hline
\end{tabular}

ATRIA, Anticoagulation and risk factors in atrial fibrillation; $\mathrm{CHADS}_{2}$ : Congestive heart failure, hypertension, age $\geq 75$ years, diabetes mellitus and stroke prior stroke or transient ischemic attack or thromboembolism (doubled); $\mathrm{CHA}_{2} \mathrm{DS}_{2}-\mathrm{VASc}$ : Congestive heart failure, hypertension, age $\geq 75$ years (doubled), diabetes mellitus, prior stroke or transient ischemic attack or thromboembolism (doubled), vascular disease, age 6574, and sex category; HAS-BLED, Hypertension, abnormal renal and liver function, stroke, bleeding, labile INRs, elderly (age $>65$ years), drugs or alcohol; INR, international normalized ratio; NVAF, non-valvular atrial fibrillation; SD, standard deviation; Std Diff, standardized difference.

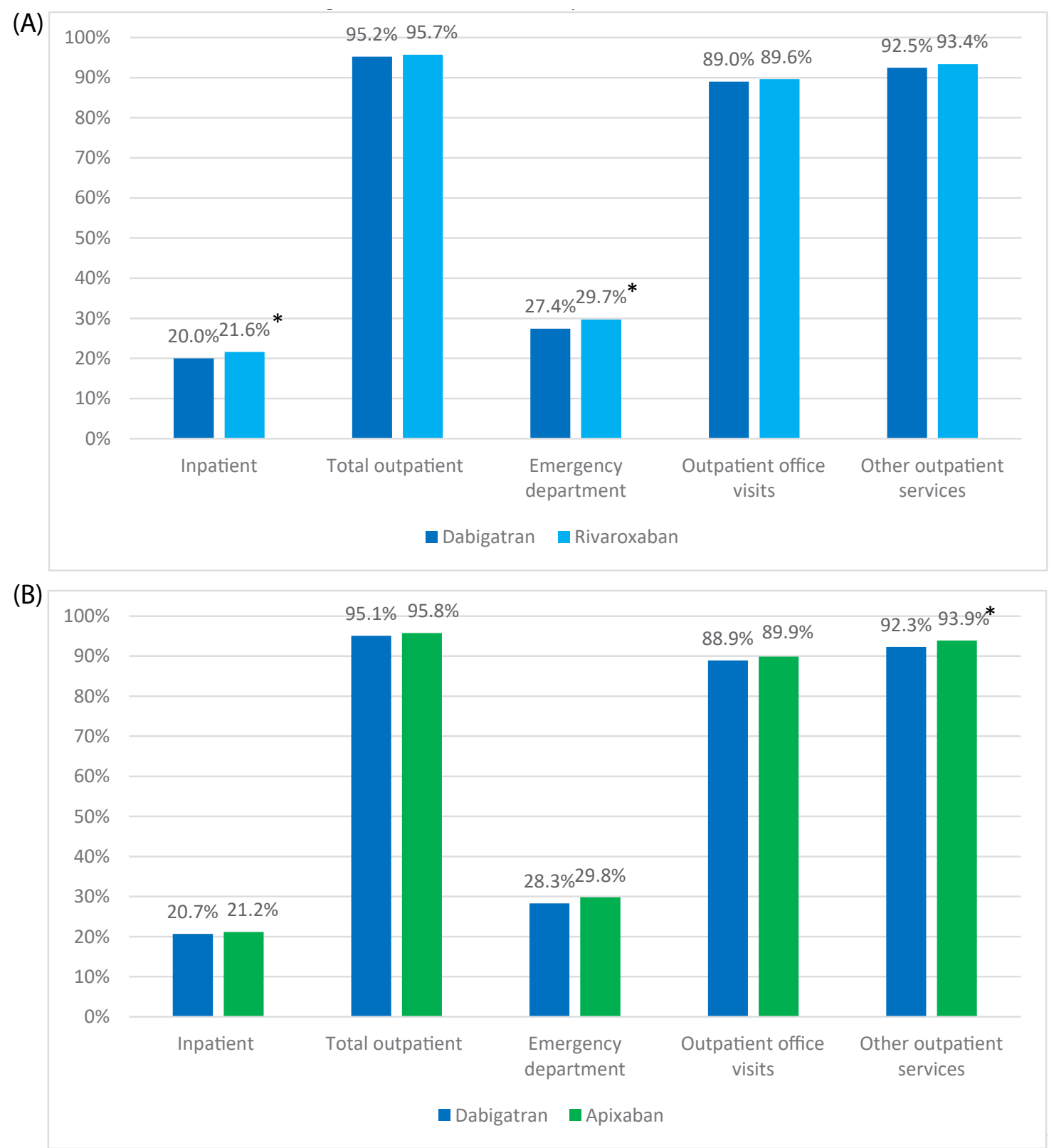

*P<0.001. $P>0.05$ for all other comparisons.

Figure 2: Percent of patients with all-cause healthcare utilization by service category

(A) Between matched dabigatran and rivaroxaban patients

(B) Between matched dabigatran and apixaban patients. 
Table 3: Healthcare resource utilization, by resource category, per patient per month

\begin{tabular}{|c|c|c|c|c|c|c|}
\hline Per patient per month utilization & Dabigatran & Apixaban & $P$ value & Dabigatran & Rivaroxaban & $P$ value \\
\hline Patient count & 5,761 & 5,761 & & 8,679 & 8,679 & \\
\hline \multicolumn{7}{|c|}{ Number of services - patients with $\geq 1$ service (mean, SD) } \\
\hline Inpatient admissions & $0.233(0.265)$ & $0.211(0.246)$ & 0.031 & $0.230(0.261)$ & $0.224(0.260)$ & 0.454 \\
\hline Average LOS per hospitalization & $1.096(2.834)$ & $0.917(1.807)$ & 0.062 & $1.103(3.120)$ & $0.999(2.473)$ & 0.269 \\
\hline Cumulative LOS (bed days) & $1.334(4.044)$ & $1.174(2.450)$ & 0.239 & $1.335(4.333)$ & $1.196(2.735)$ & 0.245 \\
\hline Outpatient visits & $3.989(4.056)$ & $4.121(4.087)$ & 0.089 & 3.865 (3.994) & $4.062(4.326)$ & 0.002 \\
\hline Emergency department visits & $0.470(0.563)$ & $0.521(0.660)$ & 0.016 & $0.477(0.641)$ & $0.517(0.624)$ & 0.026 \\
\hline Outpatient office visits & $1.205(0.900)$ & $1.212(0.922)$ & 0.708 & $1.173(0.884)$ & $1.222(0.946)$ & $<0.001$ \\
\hline Other outpatient visits & $2.806(3.536)$ & $2.879(3.415)$ & 0.279 & $2.708(3.452)$ & $2.823(3.742)$ & 0.043 \\
\hline Outpatient pharmacy claims & $4.385(2.758)$ & $4.376(2.840)$ & 0.855 & $4.256(2.667)$ & $4.249(2.782)$ & 0.873 \\
\hline Dabigatran claims & $0.734(0.319)$ & $0.299(0.311)$ & 0.006 & $0.726(0.318)$ & $0.158(0.140)$ & $<0.001$ \\
\hline Apixaban claims & $0.316(0.306)$ & $0.758(0.346)$ & $<0.001$ & $0.245(0.263)$ & $0.225(0.173)$ & 0.710 \\
\hline Rivaroxaban claims & $0.236(0.298)$ & $0.290(0.259)$ & 0.489 & $0.228(0.288)$ & $0.758(0.346)$ & $<0.001$ \\
\hline \multicolumn{7}{|l|}{ Number of services - all patients (mean, SD) } \\
\hline Inpatient admissions & $0.048(0.153)$ & $0.045(0.142)$ & 0.187 & $0.046(0.148)$ & $0.048(0.152)$ & 0.305 \\
\hline Outpatient visits & $3.794(4.048)$ & $3.949(4.085)$ & 0.042 & $3.678(3.984)$ & $3.886(4.312)$ & $<0.001$ \\
\hline Emergency department visits & $0.133(0.366)$ & $0.155(0.432)$ & 0.003 & $0.131(0.397)$ & $0.154(0.414)$ & $<0.001$ \\
\hline Outpatient office visits & $1.072(0.930)$ & $1.090(0.948)$ & 0.316 & $1.044(0.911)$ & $1.094(0.970)$ & $<0.001$ \\
\hline Other outpatient visits & $2.590(3.478)$ & $2.704(3.381)$ & 0.073 & $2.504(3.395)$ & $2.638(3.685)$ & 0.013 \\
\hline Outpatient pharmacy claims & $4.385(2.758)$ & $4.376(2.840)$ & 0.855 & $4.256(2.667)$ & $4.249(2.782)$ & 0.873 \\
\hline Dabigatran claims & $0.734(0.319)$ & $0.000(0.011)$ & $<0.001$ & $0.726(0.318)$ & $0.000(0.010)$ & $<0.001$ \\
\hline Apixaban claims & $0.001(0.019)$ & $0.758(0.346)$ & $<0.001$ & $0.001(0.016)$ & $0.002(0.024)$ & $<0.001$ \\
\hline Rivaroxaban claims & $0.003(0.040)$ & $0.001(0.022)$ & 0.004 & $0.003(0.040)$ & $0.758(0.346)$ & $<0.001$ \\
\hline
\end{tabular}

$21.9 \%$ ) or apixaban patients (22.3\% vs. $23.7 \%$ ). The time to readmission was longer for dabigatran patients compared with rivaroxaban patients ( 8.2 vs. 7.8 days, $p<0.001)$ but comparable with apixaban patients ( 8.1 vs. 8.4 days).

\section{Discussion}

This study is the first real-world data assessment comparing healthcare resource utilization among NVAF patients initially treated with warfarin who eventually switched to either dabigatran, rivaroxaban, or apixaban. This comparison is timely and important because many NVAF patients are expected to switch from warfarin to NOACs following the 2019 AHA/ACC/HRS guidelines ${ }^{15}$ that recommend NOACs over warfarin. In this retrospective database analysis of healthcare resource utilization using head-to-head comparisons of warfarin switchers initiating dabigatran vs. rivaroxaban and dabigatran vs. apixaban, we found significantly higher proportions of rivaroxaban patients with hospital and ED visits compared with dabigatran-treated patients. Use of other outpatient services was lower for dabigatran compared with both rivaroxaban and apixaban users. Overall, there were fewer outpatient visits and ED visits PPPM for dabigatran patients compared with the other two NOAC groups. In addition, the number of office visits and other outpatient visits PPPM were significantly lower for dabigatran patients compared with rivaroxaban patients, though similar when compared with apixaban.

Prior studies of healthcare resource utilization commonly compared NOACs with warfarin. Studies comparing dabigatran with warfarin patients found significantly fewer office visits (dabigatran visits ranging from 0.70 to 1.98 PPPM and warfarin visits ranging from 0.90 to $2.96 \mathrm{PPPM}^{16,17,30,31}$, significantly lower inpatient admissions (ranged $0.06-0.09$ vs. $0.07-0.10$, respectively) $)^{17,31}$, length of stay ( 4.0 vs 4.6 days) ${ }^{31}$, and ED visits $^{16,31}$. Our results were in general agreement and fitted within these ranges.

An earlier study by Gilligan et al. ${ }^{32}$ found dabigatran patients had significantly fewer inpatient admissions and ED visits, and higher numbers of outpatient visits and pharmacy claims than apixaban patients. In the same study, compared with rivaroxaban, dabigatran patients had fewer admissions, outpatient visits, and pharmacy claims, and similar ED visits compared with apixaban and rivaroxaban, as well as lower healthcare resource utilization across all service categories (with exception to LOS) when compared with warfarin patients.

This study adds to the existing head-to-head comparison literature by focusing on NVAF patients who switched to NOACs from warfarin using real-world data that cover a large range of health plans, providers, and both commercial and Medicare payers. Consistent with previous comparisons, the current results display significant evidence of lower healthcare resource utilization for dabigatran compared with rivaroxaban for hospitalizations, ED visits, and outpatient visits. Dabigatran patients also displayed 
lower numbers of ED and outpatient visits compared with matched apixaban patients. Patients, providers, and payers may find significant benefits from these lower healthcare resource intensities associated with the use of dabigatran after warfarin discontinuation. Indeed, forthcoming studies will enable finer discernment of additional resource savings that can potentially be associated with a monetary value for our healthcare systems.

\section{Limitations}

This study was subject to limitations, including those inherent with retrospective administrative healthcare claims analyses. Claims are intended to support reimbursement and therefore variables found on claims have coding limitations and possible data entry errors. NVAF was defined based on diagnosis and procedure codes, thus misclassification error is possible for NVAF, covariates, and study outcomes due to missing or inaccurate codes. Medication usage was based on filled prescriptions that patients were assumed to have taken as prescribed; however, it was unknown whether medications were actually taken. Specific information for the reasons why patients switched from warfarin to a NOAC, such as improved efficacy or diminished side effects, are not available in claims data and remain unknown. The variance around the mean time from warfarin discontinuation to NOAC initiation extended from one to several months, and it is possible that these patients may have been treated with something other than an anticoagulant prior to initiating any of the NOACs. Patients' medical and prescription history was limited to administrative claims during the reporting months in this study. Due to the large sample sizes, small differences computed as statistically significant between comparators may not have significant clinical or cost importance. There may be systematic differences between the treatment groups that account for differences found in healthcare resource utilization. While propensity score matching provided adjustment for differences between treatment groups, there is the potential for unmeasured confounders given information was limited to characteristics that could be measured using administrative claims. This study was based on commercially-insured and Medicare covered individuals in the MarketScan databases, which are convenience samples of contributing US commercial and Medicare payers. Consequently, results of this analysis may not be generalizable to other US or international populations of NVAF patients, with other insurances or without health insurance coverage. Despite these limitations, this study provides a valuable insight among patients who switched from warfarin therapy, and the resulting effects on their healthcare utilization after switching to different NOACs.

\section{Conclusions}

Warfarin treated NVAF patients switch to NOACs due to various reasons, and this switching is expected to increase per the new AHA/ACC/HRS guidelines. Although the current literature provides head-to-head comparisons on healthcare resource utilization among NOACs, there are little data on comparisons in patients who switched to NOACs from warfarin. The current study indicates that the use of dabigatran following warfarin therapy may enable significant healthcare resource utilization savings compared with those who switched to rivaroxaban or apixaban following warfarin therapy. These findings provide valuable information that may facilitate physicians' treatment decisions in guiding patients who may be candidates for switching to a NOAC from warfarin.

\section{Abbreviations}

AF: Atrial fibrillation; ATRIA: Anticoagulation and risk factors in atrial fibrillation; $\mathrm{CHADS}_{2}$ : Congestive heart failure, hypertension, age $\geq 75$ years, diabetes mellitus and stroke prior stroke or transient ischemic attack or thromboembolism (doubled); $\mathrm{CHA}_{2} \mathrm{DS}_{2}$-VASc: Congestive heart failure, hypertension, age $\geq 75$ years (doubled), diabetes mellitus, prior stroke or transient ischemic attack or thromboembolism (doubled), vascular disease, age 65-74, and sex category; CKD: Chronic kidney disease; DCI: Deyo-Charlson comorbidity index; ED: Emergency department; HAS-BLED: Hypertension, abnormal renal and liver function, stroke, bleeding, labile INRs, elderly (age > 65 years), drugs or alcohol; HCRU: Healthcare resource utilization; ICD-9-CM: International Classification of Diseases, Ninth Revision, Clinical Modification; IRB: Institutional Review Board; LOS: Length of stay (hospital inpatient); NOAC: Novel oral anticoagulant; NSAIDs: Nonsteroidal anti-inflammatory drugs; NVAF: Non-valvular atrial fibrillation; OAC: Oral anticoagulant; PPPM: Per patient per month; PSM: Propensity score matching; SD: Standard deviation; SNRIs: Serotonin-norepinephrine reuptake inhibitors; SSRIs: Selective serotonin reuptake inhibitors; TIA: Transient ischemic attack

\section{Funding}

This study was sponsored by Boehringer Ingelheim Pharmaceuticals, Inc., Ridgefield, CT, USA.

\section{Authors' Full Disclosures}

Jessica Franchino-Elder, Briain 0 Hartaigh, and Cheng Wang, are employees of Boehringer Ingelheim Pharmaceuticals. Xue Song, Caroline Henriques, and Amy Sainski-Nguyen are employees of IBM Watson Health, which received compensation from Boehringer Ingelheim Pharmaceuticals for the overall conduct of the study and preparation of the manuscript. Adrienne Gilligan was an employee of IBM Watson Health during the time of the study and writing of the manuscript. 


\section{Acknowledgements}

Editorial/medical writing support for this manuscript was provided by Jay Margolis, PharmD (IBM Watson Health).

\section{References}

1. Colilla S, Crow A, Petkun W, et al. Estimates of current and future incidence and prevalence of atrial fibrillation in the U.S. adult population. Am J Cardiol. 2013; 112: 1142-7.

2. Go AS, Hylek EM, Phillips KA, et al. Prevalence of diagnosed atrial fibrillation in adults: national implications for rhythm management and stroke prevention: the AnTicoagulation and Risk Factors in Atrial Fibrillation (ATRIA) Study. JAMA. 2001; 285: 2370-5.

3. Mozaffarian D, Benjamin EJ, Go AS, et al. Heart disease and stroke statistics--2015 update: a report from the American Heart Association. Circulation. 2015; 131: e29-322.

4. Naccarelli GV, Varker $\mathrm{H}$, Lin J, et al. Increasing prevalence of atrial fibrillation and flutter in the United States. Am J Cardiol. 2009; 104: 15349.

5. Wolf PA, Singer DE. Preventing stroke in atrial fibrillation. Am Fam Physician. 1997; 56: 2242-50.

6. National Heart, Lung, and Blood Institute. What is atrial fibrillation? [cited 2015 October 15]; Available from: http://www.nhlbi.nih.gov/ health/health-topics/topics/af.

7. Wolf PA, Abbott RD, Kannel WB. Atrial fibrillation as an independent risk factor for stroke: the Framingham Study. Stroke. 1991; 22: 983-8.

8. Stettin GD. Treatment of nonvalvular atrial fibrillation. West J Emerg Med. 1995; 162: 331-9.

9. Camm AJ, Lip GY, De Caterina R, et al. 2012 focused update of the ESC Guidelines for the management of atrial fibrillation: an update of the 2010 ESC Guidelines for the management of atrial fibrillation. Developed with the special contribution of the European Heart Rhythm Association. Eur Heart J. 2012; 33: 2719-47.

10. January CT, Wann LS, Alpert JS, et al. 2014 AHA/ACC/HRS guideline for the management of patients with atrial fibrillation: a report of the American College of Cardiology/American Heart Association Task Force on practice guidelines and the Heart Rhythm Society. Circulation. 2014; 130: e199-267.

11. Kirchhof P, Benussi S, Kotecha D, et al. 2016 ESC Guidelines for the management of atrial fibrillation developed in collaboration with EACTS. Eur J Cardiothorac Surg. 2016; 50: e1-e88.

12. Verma A, Cairns JA, Mitchell LB, et al. 2014 focused update of the Canadian Cardiovascular Society Guidelines for the management of atrial fibrillation. Can J Cardiol. 2014; 30: 1114-30.

13. Wann LS, Curtis AB, Ellenbogen KA, et al. 2011 ACCF/AHA/HRS focused update on the management of patients with atrial fibrillation (update on Dabigatran): a report of the American College of Cardiology Foundation/American Heart Association Task Force on practice guidelines. Circulation. 2011; 123: 1144-50.

14. You JJ, Singer DE, Howard PA, et al. Antithrombotic therapy for atrial fibrillation: Antithrombotic Therapy and Prevention of Thrombosis, 9th ed: American College of Chest Physicians Evidence-Based Clinical Practice Guidelines. Chest. 2012; 141: e531S-e75S.

15. January CT, Wann LS, Calkins H, et al. 2019 AHA/ACC/HRS focused update of the 2014 AHA/ACC/HRS Guideline for the management of patients with atrial fibrillation: A report of the American College of Cardiology/American Heart Association task force on clinical practice guidelines and the Heart Rhythm Society. J Am Coll Cardiol. 2019; S0735-1097(19)30209-8.

16. Bancroft T, Lim J, Wang C, et al. Health care resource utilization, costs, and persistence in patients newly diagnosed as having nonvalvular atrial fibrillation and newly treated with dabigatran versus warfarin in the United States. Clin Ther. 2016; 38: 545-56.e1-6.

17. Francis $\mathrm{K}, \mathrm{Yu} \mathrm{C}$, Alvrtsyan $\mathrm{H}$, et al. Healthcare utilization and costs associated with dabigatran compared to warfarin treatment in newly diagnosed patients with non-valvular atrial fibrillation. Current Med Res Opin. 2015; 31: 2189-95.

18. Zalesak M, Siu K, Francis K, et al. Higher persistence in newly diagnosed nonvalvular atrial fibrillation patients treated with dabigatran versus warfarin. Circ Cardiovasc Qual Outcomes. 2013; 6: 567-74.

19. Connolly SJ, Ezekowitz MD, Yusuf S, et al. Dabigatran versus warfarin in patients with atrial fibrillation. N Engl J Med. 2009; 361: 1139-51.

20. Diener HC, Aisenberg J, Ansell J, et al. Choosing a particular oral anticoagulant and dose for stroke prevention in individual patients with non-valvular atrial fibrillation: part 2. Eur Heart J. 2017; 38:v860-8.

21. Verdecchia P, Angeli F, Aita A, et al. Why switch from warfarin to NOACs? Intern Emerg Med. 2016; 11: 289-93.

22. Farr AM, Johnston SS, Ritchings C, et al. Persistence, adherence, and all-cause healthcare costs in atazanavir- and darunavir-treated patients with human immunodeficiency virus in a real-world setting. J Med Econ. 2016; 19: 386-96.

23. Hao Y, Li N, Fang AP, et al. Real-world analysis of medical costs and healthcare resource utilization in elderly women with HR+/HER2metastatic breast cancer receiving everolimus-based therapy or chemotherapy. Adv Ther. 2016; 33: 983-97.

24. Mucha L, Masia NA, Axelsen KJ. Per-patient-per-month drug costs in Medicare Part D protected classes. Pharmacoeconomics. 2006; 24 Suppl 3: 79-84.

25. Johnson BH, Smoyer-Tomic KE, Siu K, et al. Redmission among hospitalized patients with non-nalvular atrial fibrillation. Am J Health Syst Pharm. 2013; 70: 414-22.

26. Deyo RA, Cherkin DC, Ciol MA. Adapting a clinical comorbidity index for use with ICD-9-CM administrative databases. J Clin Epidemiol. 1992; 45: 613-9.

27. Gage BF, Waterman AD, Shannon W, et al. Validation of clinical classification schemes for predicting stroke: results from the National Registry of Atrial Fibrillation. JAMA. 2001; 285: 2864-70.

28. Lip GY, Frison L, Halperin JL, et al. Comparative validation of a novel risk score for predicting bleeding risk in anticoagulated patients with atrial fibrillation: the HAS-BLED (Hypertension, Abnormal Renal/ Liver Function, Stroke, Bleeding History or Predisposition, Labile INR, Elderly, Drugs/Alcohol Concomitantly) score. J Am Coll Cardiol. 2011; 57: 173-80.

29. Centers for Medicare and Medicaid Services. High-risk medication risk-priority health 2016. [cited 2016 March 28]; Available from: https://www.priorityhealth.com/provider/clinical-resources/ medication-resources/ /media/documents/pharmacy/cms-highrisk-medications.pdf.

30. Fu A, Jain R, Sander SD, et al. Healthcare resource utilization and costs among patients with non-valvular atrial fibrillation using dabigatran or warfarin for stroke prevention. Quality of Care and Outcomes Research in Cardiovascular Disease and Stroke (QCOR); Baltimore, Maryland2014.

31. Sussman M, Ghate S, Sutherland SP, et al. Resource use among nonvalvular atrial fibrillation patients. Am J Pharm Benefits. 2016; 8: 84-92.

32. Gilligan AM, Franchino-Elder J, Song X, et al. Comparison of all-cause costs and healthcare resource use among patients with newlydiagnosed non-valvular atrial fibrillation newly treated with oral anticoagulants. Curr Med Res Opin. 2018; 34: 285-95. 